\title{
Screening for foot-and-mouth disease virus in livestock- wildlife interface areas of Tanzania
}

\author{
Authors: \\ Emma Peter ${ }^{1}$ \\ Christopher J. Kasanga ${ }^{1}$ \\ Raphael Sallu ${ }^{2}$ \\ Mkama Mathias ${ }^{2}$ \\ Mmeta Yongolo² \\ Misheck Mulumba ${ }^{3}$ \\ Ezekia Ranga ${ }^{4}$ \\ Philemon N. Wambura ${ }^{1}$ \\ Mark M. Rweyemamu \\ Affiliations: \\ ${ }^{1}$ Southern African Centre \\ for Infectious Disease \\ Surveillance, Sokoine \\ University of Agriculture, \\ Tanzania \\ ${ }^{2}$ Tanzania Veterinary \\ Laboratory Agency, Tanzania \\ ${ }^{3}$ Southern African \\ Development Community \\ Secretariat, Botswana \\ ${ }^{4}$ Ministry of Livestock \\ Development and Fisheries, \\ Tanzania \\ Correspondence to: \\ Christopher Kasanga \\ Email: \\ christopher.kasanga@sacids. \\ org \\ Postal address: \\ PO Box 3019, Chuo Kikuu, \\ Morogoro, Tanzania \\ How to cite this article: \\ Peter, E., Kasanga, C.J., \\ Sallu, R., Mathias, M., \\ Yongolo, M., Mulumba, M. \\ et al., 2014, 'Screening for \\ foot-and-mouth disease \\ virus in livestock-wildlife \\ interface areas of Tanzania', \\ Onderstepoort Journal of \\ Veterinary Research 81(2), \\ Art. \#723, 1 page. http:// \\ dx.doi.org/10.4102/ojvr. \\ v81i2.723 mouth disease (FMD) virus (FMDV) in animals. In this study, the singleplex real-time RT-PCR (qRT-PCR) assay employing the Callahan 3DF-2, 3DF-R primers and Callahan 3DP-1 probe was used in screening for FMDV genome on oesophageal-pharyngeal (OP) fluids. The OP samples were collected from cattle and African buffaloes in livestock-wildlife interface areas of Mikumi, Mkomazi and Ruaha National Parks in Tanzania in 2011, which included National Parks and the surrounding areas. The detection rates of FMDV genome were 5.88\% $(n=3), 19.44 \%(n=7)$ and 41.18\% $(n=21)$ in Mkomazi, Ruaha and Mikumi National Parks, respectively. FMDV detection rates in Ruaha and Mikumi were significantly higher in the African buffaloes $(p<0.05)$ compared to those in cattle. There was no correlation of FMDV detection with either age or sex of the animals in the three National Parks. These findings indicate that cattle and buffaloes in Mikumi, Ruaha and Mkomazi were naturally infected with FMDV. Furthermore, the higher FMDV detection rates in buffaloes suggest that buffaloes could potentially act as reservoirs for FMDV and possibly play a significant role in transmission of the virus to other in-contact susceptible animals. Further studies including serotyping, virus isolation, experimental infection and sequencing of the viruses, are required to elucidate the complex epidemiology of FMD in cattle and buffaloes in the livestock-wildlife interface areas in Tanzania.

\section{Acknowledgements}

This work was supported by the Wellcome Trust Grant WT087546MA to the Southern African Centre for Infectious Diseases \& Surveillance (SACIDS). 